\title{
Predicting Radioresistant Biomarkers in Nasopharyngeal Carcinoma Patients Via Protein- Protein Interaction Network Analysis
}

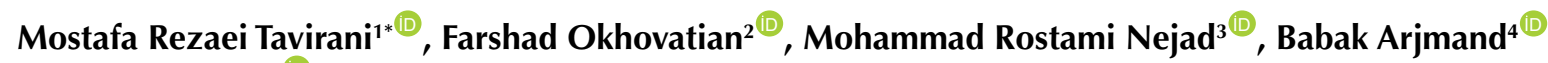 \\ , Zahra Razzaghis ${ }^{\circledR}$ \\ ${ }^{1}$ Proteomics Research Center, Faculty of Paramedical Sciences, Shahid Beheshti University of Medical Sciences, Tehran, \\ Iran. \\ ${ }^{2}$ Physiotherapy Research Center, School of Rehabilitation, Shahid Beheshti University of Medical Sciences, Tehran, Iran \\ ${ }^{3}$ Research Institute for Gastroenterology and Liver Diseases, Gastroenterology and Liver Diseases Research Center, \\ Shahid Beheshti University of Medical Sciences, Tehran, Iran. \\ ${ }^{4}$ Cell Therapy and Regenerative Medicine Research Center, Endocrinology and Metabolism Molecular-Cellular Sciences \\ Institute, Tehran University of Medical Sciences, Tehran, Iran. \\ ${ }^{5}$ Laser Application in Medical Sciences Research Center, Shahid Beheshti University of Medical Sciences, Tehran, Iran.
}

\section{*Correspondence to Mostafa Rezaei Tavirani, Email tavirany@yahoo.com}

\section{Received: July 28, 2021} Accepted: September 8, 2021 Published online December 1 , 2021

\begin{abstract}
Introduction: Radiotherapy as the first-line nasopharyngeal carcinoma (NPC) treatment provides different responses including radioresistant and radiosensitive states. In order to investigate the molecular basis of radioresistancy, protein-protein interaction network analysis of proteome data prior to therapy was performed.

Methods: 20 dysregulated proteins of the patients who were radioresistant were extracted from the literature. Cytoscape and its plug-ins were used for the resistant network construction and its centrality analysis. Furthermore, ClueGO+ CluePedia application determined the most statistically significant biological processes (BP) related to the hubs.

Results: Fourteen hubs were concluded and no differentially expressed protein (DEP) was among these agents. Among the hubs, albumin (ALB) and fibronectin (FN1) were the hub-bottlenecks, and the Serpin family was present. What is more, SERPIND1 was the highest degree-valued DEP in the network.

Conclusion: It can be concluded that the central elements of the NPC network could be noteworthy for improving the radiotherapy outcome and overcoming its limitations. However, complementary studies are required for a better understanding of their major role.

Keywords: Nasopharyngeal carcinoma; Radiotherapy; Radioresistance; Biomarkers; Protein-protein interaction network analysis.
\end{abstract}

\section{Introduction}

Nasopharyngeal carcinoma (NPC), developing in the nasopharynx, is endemic in Southeast Asia and recognized as a head and neck malignancy. ${ }^{1}$ The World Health Organization (WHO) grouped NPC into keratinizing squamous cell carcinoma, non-keratinizing squamous carcinoma, and undifferentiated carcinoma subtypes. ${ }^{2}$ Risk factors associated with NPC are genetics, Epstein-Barr Virus(EBV), dietary factors, and smoking., Additionally, this type of complex cancer is very malignant and heterogenic on a molecular basis. ${ }^{5}$ It can progress from lymphatic nodes to other organs even in the early stages of the disease. ${ }^{6}$ Therefore, early detection of this type of cancer is vital for increasing the survival rate by applying proper treatment approaches. ${ }^{7}$ Some of the options of early detection are MRI and endoscopy. ${ }^{8}$ In terms of treatment options, National Comprehensive Cancer Network suggests such common methods as combined radiotherapy and chemotherapy. ${ }^{9}$ The first line treatment protocol in this type of cancer is radiotherapy that induces apoptosis in cancer cells. There are different forms of radiation therapy such as 2D-radiotherapy, 3D-radiotherapy, and intensity-modulated radiotherapy. ${ }^{10}$ Patients, including radiosensitive and radioresistant individuals, respond differently to this type of treatment due to variations in a molecular basis. ${ }^{6}$ To establish this difference, molecular studies could be the answer. For instance, previous studies highlighted that metabolic processes have associations with radiotherapy resistance. ${ }^{11}$ Proteomics as one of the molecular approaches is very promising in detecting biomarkers in the subject of the study. Further analysis of differentially expressed proteins (DEPs) in a network

Please cite this article as follows: Rezaei Tavirani M, Okhovatian F, Rostami Nejad M, Arjmand B, Razzaghi Z. Predicting radioresistant biomarkers in nasopharyngeal carcinoma patients via protein-protein interaction network analysis. J Lasers Med Sci. 2021;12:e76. doi:10.34172/jlms.2021.76. 
pattern can offer more potential biomarkers considering their crucial role on a network basis. ${ }^{12}$ Centrality analysis is mostly done by considering two common parameters of degree and betweenness values. Nodes with the highest values of these two parameters are considered network stability fundamentals. ${ }^{13}$ In this study, to evaluate radioresistance underlying the mechanisms of NPC, the network interaction analysis of DEPs in serum between radiosensitive and radioresistant patients was executed. It can provide the critical possible drug targets in the radiotherapy of the patients.

\section{Materials and Methods}

Data of an investigation about NPC patients (male and female) without any previous types of treatments which were selected for radiotherapy ${ }^{6}$ was analyzed. These patients were diagnosed at either stage $\amalg$ or IV, and their protein samples were provided from serum prior to the designated treatment. The patients were exposed to 70.4 Gy/2.2 Gy over 32 fractions [PGTVnx], 70.4 Gy/2.2Gy over 32 fractions [PGTVnd], $60.8 \mathrm{~Gy} / 1.9$ Gy over 32 fractions [PTV1], and $54 \mathrm{~Gy} / 1.8$ Gy over 30 fractions [PTV2]), and based on their response to treatment, they were grouped into two categories: radiosensitive and radioresistant groups. Among the determined proteins via quantitative label-free proteomics, 20 of them were altered in expression between the groups of radiosensitive and radioresistant individuals. That is, among these proteins, 8 of them were up-regulated and 12 of them were downregulated. More details of the methods are described in the original document. ${ }^{6}$ These proteins were subjected to a protein-protein interaction network analysis, and two networks via the Cytoscape platform (https://cytoscape. org/) were obtained. The first one contains only the 20 DEPs

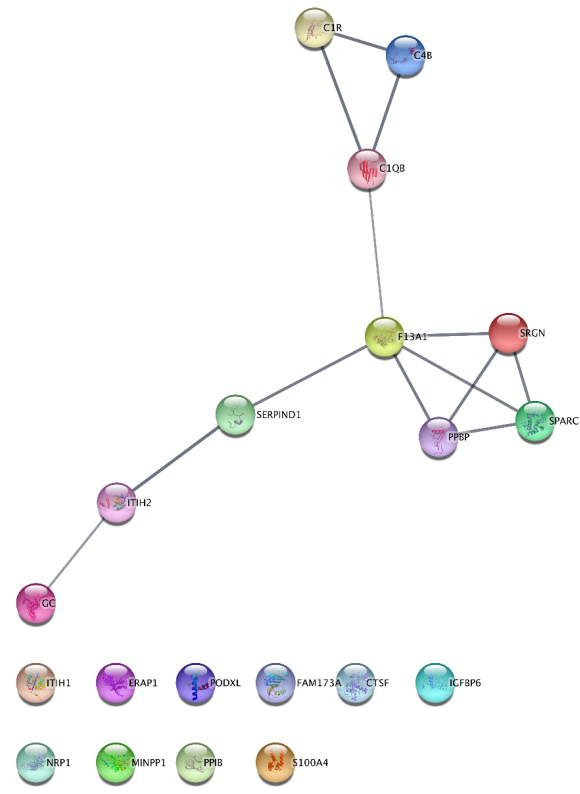

Figure 1. Network Visualization of Query Proteins. The number of nodes: 20 , the number of links: 13 , and the confidence score cutoff $\geq 0.5$ while the second one has additional proteins surrounding these query proteins. String db (http://string-db.org/) is the application in Cytoscape, and the network query and mapping could be conducted through this source. ${ }^{14}$ The confidence score cutoff for both network constructions was set to 0.04 as the default option. Furthermore, centrality analysis based on degree and betweenness centrality was carried out by NetworkAnalyzer. ${ }^{15}$ A node showing the highest numbers of edges acts as a hub agent that is important for the network strength. The hub identification is calculated based on $20 \%$ of the highest values of the degree parameter. ${ }^{16}$ The hubs were annotated through a combined plug-in of ClueGO 2.5.8+CluePedia 1.5.8 for biological processes (BP). ${ }^{17,18}$ This search tool identifies different groups of $\mathrm{BP}$ which are related to the query hubs. The value of $P \leq 0.01$ was designated for identifying statistically significant groups of highlighted BP terms. The number of genes linked to each group was a minimum of 2 and the percentage of gene contribution in each group was set to 3. The kappa score cutoff for the grouping was also set to 0.5 which was higher than the default option. Moreover, the Bonferroni step-down test was the corrected $P$ value method.

\section{Results}

The interaction network analysis of radioresistant proteins of NPC was retrieved via the String db plug-in, Cytoscape, as shown in Figures 1 and 2.

The first network is composed of only the main query proteins, which indicates that half of the NPC radioresistant proteins are not in direct connections. These proteins are ITIH1, ERAP1, PODXL, FAM173A, CTSF, IGFBP6, NRP1, MINPP1, PPIB, and S100A4.

To construct a network of differentially expressed NPC

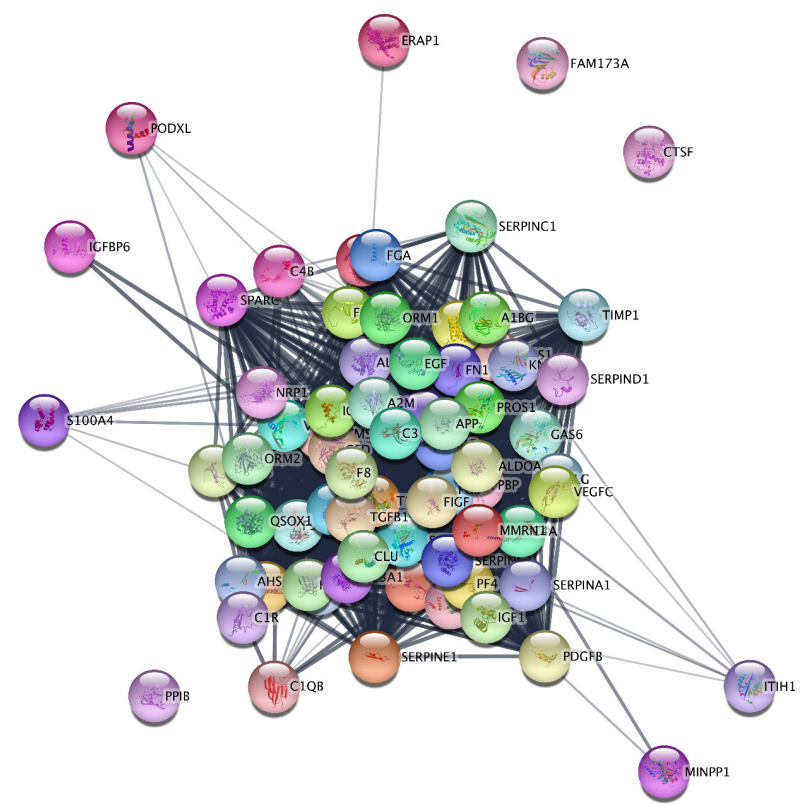

Figure2. A Network of NPC Radioresistance Proteins and Added Nodes. The number of nodes: 70, the number of links: 1470 , and the confidence score cutoff $\geq 0.5$ 
proteins with surrounding proteins, 50 nodes were added in Figure 2.

The pattern of the second network shows three query proteins are isolated due to add 50 proteins into the network. These nodes are PPIB, CTSF, and FAM173A.

To detect the highest valued nodes of the PPI network, $20 \%$ of the highest amounts of degree calculated by NetworkAnalyzer were determined as listed in Table 1.

ALB and FN1 are the highest valued nodes in this PPI network with degrees of 60 and 59, and betweenness centrality values of 0.05 and 0.02 respectively. In fact, ALB has the highest degree in this analysis and is the hubbottleneck. The rest of the nodes indicate low amounts of BC, which are 0.01. ALB and FN1 are called the hubbottlenecks of the interaction network.

None of the nodes listed in Table 1 are from query proteins known as differentially expressed ones. The

Table 1. The list of Nodes of the PPI Network With Degree (K) and Betweenness Centrality (BC) Ranked Based on Degree Values

\begin{tabular}{llll}
\hline Row & Display Name & K & BC \\
\hline 1 & ALB & 60 & 0.05 \\
2 & FN1 & 59 & 0.02 \\
3 & SERPINA1 & 58 & 0.01 \\
4 & KNG1 & 58 & 0.01 \\
5 & SERPING1 & 57 & 0.01 \\
6 & HRG & 57 & 0.01 \\
7 & VWF & 57 & 0.01 \\
8 & AHSG & 57 & 0.01 \\
9 & FGA & 57 & 0.01 \\
10 & PLG & 57 & 0.01 \\
11 & SERPINF2 & 56 & 0.01 \\
12 & TIMP1 & 56 & 0.01 \\
13 & F5 & 56 & 0.01 \\
14 & FGG & 56 & 0.01 \\
\hline
\end{tabular}

highest valued query proteins are F13A1 and PPBP with the highest degree of 52 and betweenness centrality of 0.01 . The rest of the query proteins have the lowest amount of these parameters.

To analyze the gene ontology of hubs with regard to BP, identification was handled by ClueGO + CluePedia in Figure 3.

\section{Discussion}

Radioresistance response is a dilemma in cancer treatments for patients of NPC. Understanding molecular mechanisms by which some NPC patients are radioresistant could be achieved through bioinformatics analysis of proteome data. Proteins that are differentially expressed in proteomics studies are searched for interaction network analysis. Thus, the key proteins can be obtained by centrality determination considering degree and betweenness parameters. Based on centrality analysis, none of the DEPS of NPC patients was among the hubs of the network. ALB and FN1 are the hub-bottlenecks of the PPI network as they have the highest amounts of degree and betweenness centrality. These two nodes may indicate the most promising agents of network stability in the NPC network. However, further investigation is required to link the relations. A literature survey of the hub-bottlenecks can provide better recognition of these elements in NPC risks and treatments. It has been reported that albumin (ALB) has prognostic values in NPC patients without any treatments ${ }^{19}$. The next protein is FN1 (fibronectin) that has been suggested as a radioresistant marker of head and neck squamous cell carcinoma. ${ }^{20}$ Likewise, it may also function as a radioresistant factor in NPC.

On the other hand, the Serpins family (serpin peptidase inhibitor) including SERPINA1, SERPING1, and SERPINF2 are dispersed as the hubs in this network. In fact, Serpins, in general, are connected to cancer survival. ${ }^{21,22}$ Previous studies specify that how introduced

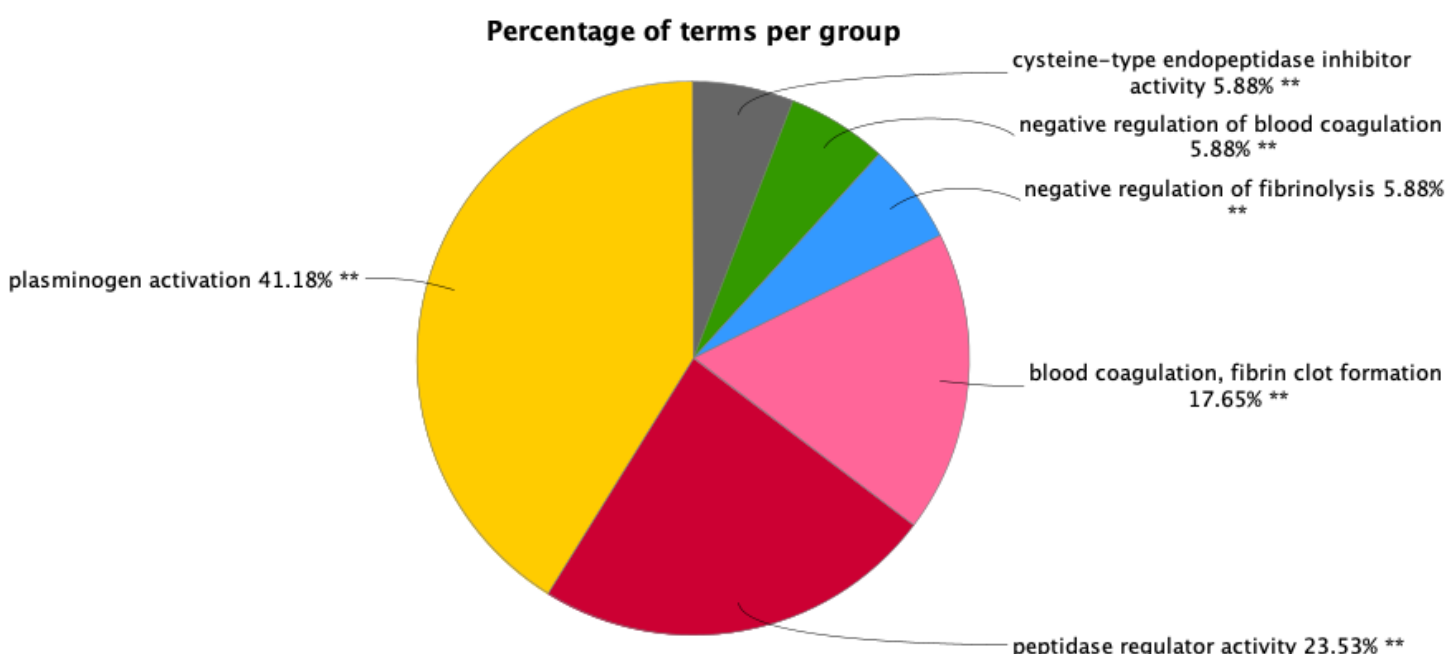

Figure3. Pie Chart View of Biological Processes in Hubs of NPC Network. The two-star sign shows $P \leq 0.01$. Plasminogen activation, peptidase regulator activity, blood coagulation, fibrin clot formation, negative regulation of fibrinolysis, negative regulation of blood coagulation, and cysteine-type endopeptidase inhibitor are the processes that contribute to the hubs. The first one is the most highlighted one since it has the highest number of terms. 
serpins act in different cancer types. SERPINA1 as serpin family A member 1 is novel in different types of cancer progression, including breast cancer, colon cancer, and gastric cancer. ${ }^{23-25}$ The next one is SERPING1 (Serpin Family G Member 1) whose low expression contributes to the prostate cancer risk. ${ }^{26}$ The last serpin family member is SERPINF2 (serpin family F member 2) which could show some association with hepatocellular carcinoma. ${ }^{27}$ None of the hub serpins was directly reported for NPC based on the literature search. SERPIND1 is the query protein whose centrality values based on designated parameters of degree and betweenness centrality in the network are 29 and 0.000369 . This protein has also shown some relation to ovarian cancer. ${ }^{28,29}$ Investigations indicates that Serpinb3a-/- mice that is equal to SERPINB3 and SERPINB4 in humans has shown resistance in radiotherapy. ${ }^{30,31}$ In addition, suppression of TP53 is also processed by the activation of TRIM21-SERPINB5 that consequently results in NPC radioresistance. ${ }^{32}$ Finally, SERPIND1 (serpin family D member 1) was also found as an up-regulated protein in the serum of radioresistant patients of NPC. ${ }^{6}$ This expresses that serpins may also have a potential role in the radioresistant manner of NPC cases.

In the next step, the gene ontology evaluation of this network shows that central nodes are participants of six BP. All of the groups are statistically significant, and the leading group is plasminogen activation. Consequently, dysregulation of these BPs could be conducted in the NPC-resistant risk.

The results show that the topology of the concluded network is in a way that fewer query proteins are involved in the central part of the network. This finding implies the contribution of additional proteins rather than DEPs in the resistance type of NPC network. That is, these hubs may also have essential roles in NPC pathogenicity, if not more valuable. FN1 as the hub-bottleneck especially indicates previous associations in radioresistant types of head and neck squamous cell carcinoma.

\section{Conclusion}

In summary, the hub agents of the network such as ALB, FN1, and Serpin members may be prominent in the differentiation of radioresistant NPC patients and radiosensitive ones. However, more analysis is essential to claim this finding for improving the therapeutic outcome of radiotherapy.

\section{Ethical considerations}

Not applicable.

\section{Conflict of Interests}

There is no conflict of interests

\section{Acknowledgments}

This article is supported by Shahid Beheshti University of

\section{Medical Sciences.}

\section{References}

1. Ding R-B, Chen P, Rajendran BK, Lyu X, Wang H, Bao J, et al. Molecular landscape and subtype-specific therapeutic response of nasopharyngeal carcinoma revealed by integrative pharmacogenomics. Nat Commun. 2021;12(1):1-19. doi: 10.1038/s41467-021-23379-3.

2. Xiao Z, Li M, Li G, Fu Y, Peng F, Chen Y, et al. Proteomic characterization reveals a molecular portrait of nasopharyngeal carcinoma differentiation. $J$ Cancer. 2017;8(4):570-7. doi: 10.7150/jca.17414.

3. Chen C, Liang K, Chang Y, Wang Y, Hsieh T, Hsu M-M, et al. Multiple risk factors of nasopharyngeal carcinoma: Epstein-Barr virus, malarial infection, cigarette smoking and familial tendency. Anticancer Res. 1990;10(2B):547-53.

4. Zheng Y, Tuppin P, Hubert A, Jeannel D, Pan Y, Zeng Y, et al. Environmental and dietary risk factors for nasopharyngeal carcinoma: a case-control study in Zangwu County, Guangxi, China. British J Cancer. 1994;69(3):508-14. doi: 10.1038/bjc.1994.92.

5. Zhao L, Fong AH, Liu N, Cho WC. Molecular subtyping of nasopharyngeal carcinoma (NPC) and a microRNA-based prognostic model for distant metastasis. J Biomed Sci. 2018;25(1):1-12. doi: 10.1186/s12929-018-0417-5.

6. Zhang G, Zhang K, Li C, Li Y, Li Z, Li N, et al. Serum proteomics identify potential biomarkers for nasopharyngeal carcinoma sensitivity to radiotherapy. Biosci Rep. 2019;39(5): BSR20190027. doi: 10.1042/ BSR20190027.

7. Hutajulu SH, Indrasari SR, Indrawati LP, Harijadi A, Duin S, Haryana SM, et al. Epigenetic markers for early detection of nasopharyngeal carcinoma in a high risk population. Mol Cancer. 2011;10(1):1-9. doi: 10.1186/1476-4598-10-48.

8. King A, Woo J, Ai Q, Chan J, Lam W, Tse I, et al. Complementary roles of MRI and endoscopic examination in the early detection of nasopharyngeal carcinoma. Ann Oncol. 2019;30(6):977-82. doi: 10.1093/annonc/mdz106.

9. Wang M-Y, Qi B, Wang F, Lin Z-R, Li M-Y, Yin W-J, et al. PBK phosphorylates MSL1 to elicit epigenetic modulation of CD276 in nasopharyngeal carcinoma. Oncogenesis. 2021;10(1):1-14. doi: 10.1038/s41389-020-00293-9. Erratum in: Oncogenesis. 2021;10(4):35.

10. Moon SH, Cho KH, Lee C-G, Keum KC, Kim Y-S, Wu $\mathrm{H}-\mathrm{G}$, et al. IMRT vs. 2D-radiotherapy or 3D-conformal radiotherapy of nasopharyngeal carcinoma. Strahlenther Onkol. 2016;192(6):377-85. doi: 10.1007/s00066-0160959-y.

11. Tang L, Wei F, Wu Y, He Y, Shi L, Xiong F, et al. Role of metabolism in cancer cell radioresistance and radiosensitization methods. J Exp Clin Cancer Res. 2018;37(1):1-15. doi: 10.1186/s13046-018-0758-7.

12. Zamanian-Azodi M, Rezaei-Tavirani M, Esmaeili S, Arjmand B, Jahani-Sherafat S. Evaluation of anticancer effect of ghost pepper: a bioinformatics assessment. Res J Pharmacogn. 2021;8(3):77-82. doi: 10.22127/ RJP.2021.267225.1666.

13. Azodi MZ, Razzaghi M, Malekpour H, Rezaei-Tavirani M, Rezaei-Tavirani M, Heidari MH. Highlighted role of "IL17 signaling pathway" in gastroesophageal reflux disease. Gastroenterol Hepatol Bed Bench. 2020;13(Suppl1):S68-74. 
doi:10.22037/ghfbb.v13i1.2245

14. Szklarczyk D, Gable AL, Lyon D, Junge A, Wyder S, HuertaCepas J, et al. STRING v11: protein-protein association networks with increased coverage, supporting functional discovery in genome-wide experimental datasets. Nucleic Acids Res. 2019;47(D1):D607-D13. doi: 10.1093/nar/ gky1131.

15. Assenov Y, Ramírez F, Schelhorn S-E, Lengauer T, Albrecht M. Computing topological parameters of biological networks. Bioinformatics. 2008;24(2):282-4. doi: 10.1093/ bioinformatics/btm554.

16. Zamanian-Azodi M, Arjmand B, Razzaghi M, Tavirani MR, Ahmadzadeh A, Rostaminejad M. Platelet and Haemostasis are the Main Targets in Severe Cases of COVID-19 Infection; a System Biology Study. Arch Acad Emerg Med. 2021;9(1): e27. doi: 10.22037/aaem.v9i1.1108.

17. Bindea G, Mlecnik B, Hackl H, Charoentong P, Tosolini M, Kirilovsky A, et al. ClueGO: a Cytoscape plug-in to decipher functionally grouped gene ontology and pathway annotation networks. Bioinformatics. 2009;25(8):1091-3. doi: 10.1093/bioinformatics/btp101.

18. Bindea G, Galon J, Mlecnik B. CluePedia Cytoscape plugin: pathway insights using integrated experimental and in silico data. Bioinformatics. 2013;29(5):661-3. doi: 10.1093/ bioinformatics/btt019.

19. Du XJ, Tang LL, Mao YP, Sun Y, Zeng MS, Kang TB et al. The pretreatment albumin to globulin ratio has predictive value for long-term mortality in nasopharyngeal carcinoma. PloS one. 2014;9(4):e94473. doi: 10.1371/journal.pone.0094473.

20. Jerhammar F, Ceder R, Garvin S, Grénman R, Grafström RC, Roberg K. Fibronectin 1 is a potential biomarker for radioresistance in head and neck squamous cell carcinoma. Cancer Biol Ther. 2010;10(12):1244-51. doi: 10.4161/ cbt.10.12.13432. Ep

21. Valiente M, Obenauf AC, Jin X, Chen Q, Zhang XH-F, Lee DJ, et al. Serpins promote cancer cell survival and vascular co-option in brain metastasis. Cell. 2014;156(5):1002-16. doi: 10.1016/j.cell.2014.01.040.

22. Wojtukiewicz M, Rucinska M, Kloczko J, Dib A, Galar M. Profiles of plasma serpins in patients with advanced malignant melanoma, gastric cancer and breast cancer. Haemostasis. 1998;28(1):7-13. doi: 10.1159/000022376.

23. Chan HJ, Li H, Liu Z, Yuan Y-C, Mortimer J, Chen S. SERPINA1 is a direct estrogen receptor target gene and a predictor of survival in breast cancer patients. Oncotarget.
2015;6(28):25815-27. doi: 10.18632/oncotarget.4441.

24. Yang J, Xiong X, Wang X, Guo B, He K, Huang C. Identification of peptide regions of SERPINA1 and ENOSF1 and their protein expression as potential serum biomarkers for gastric cancer. Tumor Biol. 2015;36(7):510918. doi: 10.1007/s13277-015-3163-2.

25. Kwon CH, Park HJ, Choi JH, Lee JR, Kim HK, Jo H-j, et al. Snail and serpinAl promote tumor progression and predict prognosis in colorectal cancer. Oncotarget. 2015;6(24):20312-26. doi: 10.18632/oncotarget.3964.

26. Peng S, Du T, Wu W, Chen X, Lai Y, Zhu D, et al. Decreased expression of serine protease inhibitor family G1 (SERPING1) in prostate cancer can help distinguish highrisk prostate cancer and predicts malignant progression. Urol Oncol. 2018;36(8):366.e1-e9. doi: 10.1016/j. urolonc.2018.05.021.

27. Chan KY, Lai PB, Squire JA, Beheshti B, Wong NL, Sy SM, et al. Positional expression profiling indicates candidate genes in deletion hotspots of hepatocellular carcinoma. Mod Pathol. 2006;19(12):1546-54. doi: 10.1038/ modpathol.3800674.

28. Zhu L, Guo Q, Jin S, Feng H, Zhuang H, Liu C, et al. Analysis of the gene expression profile in response to human epididymis protein 4 in epithelial ovarian cancer cells. Oncol Rep. 2016;36(3):1592-604. doi: 10.3892/ or.2016.4926.

29. Guo Q, Zhu L, Wang C, Wang S, Nie X, Liu J, et al. SERPIND1 affects the malignant biological behavior of epithelial ovarian cancer via the PI3K/AKT pathway: a mechanistic study. Front Oncol. 2019;9:954. doi: 10.3389/ fonc.2019.00954.

30. Thermozier S, Zhang X, Hou W, Fisher R, Epperly MW, Liu B, et al. Radioresistance of Serpinb3a-/- mice and derived hematopoietic and marrow stromal cell lines. Radiat Res. 2019;192(3):267-81. doi: 10.1667/RR15379.1.

31. Thermozier S, Zhang X, Hou W, Fisher R, Epperly MW, Liu B, Bahar I, Wang H, Greenberger JS. Radioresistance of Serpinb3a-/- Mice and Derived Hematopoietic and Marrow Stromal Cell Lines. Radiat Res. 2019;192(3):267281. doi: 10.1667/RR15379.1.

32. Zhang P, Li X, He Q, Zhang L, Song K, Yang X, et al. TRIM21-SERPINB5 aids GMPS repression to protect nasopharyngeal carcinoma cells from radiation-induced apoptosis. J Biomed Sci. 2020;27(1):1-11. doi: 10.1186/ s12929-020-0625-7. 